\title{
Simplifications in 3D High-Low Frequency Models of Multi-winding Magnetic Components (EE \& Toroidal Cores)
}

\section{J.R. González*, Roberto Prieto and Rafael Asensi \\ División de Ingeniería Electrónica, Universidad Politécnica de Madrid, Spain}

\begin{abstract}
Not every magnetic component can be simulated with 3D finite elements tools. Nowadays, there are hard difficulties to simulate 3D models because of computer limitations and the 2D models have not enough accuracy $[1,2]$.

The proposed method will simulate the magnetic component ( $E E$ and Toroidal core) in 3D and decrease the needed processing time. The method consists of several simplifications and adjustments to determine the sought parameters (resistance \& inductance) in low/high frequency without losing accuracy when compared with the real 3D model simulation.
\end{abstract}

\section{Keywords}

DC power conversion, finite-element methods, inductors, modelling, voltage transformers, 3 dimensions

\section{Introduction}

Currently, there are computer limitations that make impossible to process 3D magnetic component models with Finite Elements Analysis (FEA). In fact, it is necessary to make simplifications in the model to process the model.

Sometimes, the $2 \mathrm{D}$ model of the magnetic component is not appropriate due to the asymmetry of the component [3].

Therefore, a method with 3D simplifications has been developed to obtain the sought parameters of resistance and inductance with a similar accuracy to the real 3D model and to decrease the simulated time of the FEA program.

There are several assumptions, methods and models, aided program to simulate different components with more or less accuracy [4-13], but not with the final objective for this manuscript.

\section{Finite Elements Study}

In any $3 \mathrm{D}$ simulation, the main factor that limits the study of the component is the number of Finite Elements (FE). Most magnetic component models are composed of air, core and coils.

In determining the number of FE, the coils are the most important component. The number of coils, the shape, distance among them and the geometry can significantly impact the number of Finite Elements [14].

Among these factors, the critical one is the coil section. A study in detail has been developed in Maxwell to know more precisely how the number of FE varies with the cross-sectional shape of the coil.

The same model (EE core) has been processed several times while changing the coil cross-section (number of edges in the section). A similar study was developed using a Toroidal core. Figure 1 is obtained results. Conclusion for this study is:

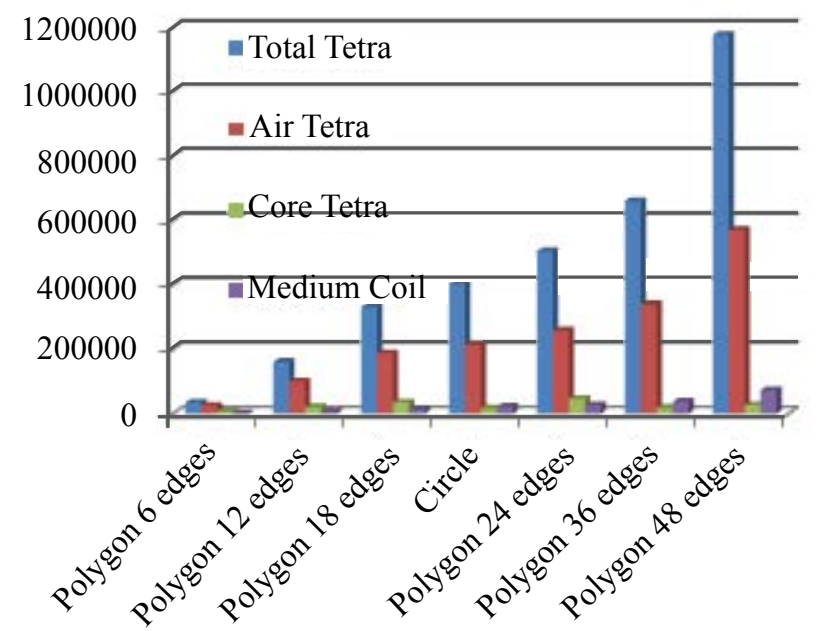

Figure 1: Comparison of number of FE among coils of different crosssections (EE core).

*Corresponding author: J.R. González, División de Ingeniería Electrónica, Universidad Politécnica de Madrid, 28006, Madrid, Spain, E-mail: jordirgt@hotmail.com

Received: September 30, 2015: Accepted: December 29, 2015: Published: December 31, 2015

Copyright: @ 2015 González JR. This is an open-access article distributed under the terms of the Creative Commons Attribution License, which permits unrestricted use, distribution, and reproduction in any medium, provided the original author and source are credited. 
1. The number of FE increases with the number of edges. The only exception is a coil with a circular cross-section where the number of $\mathrm{FE}$ is between 18 to 24 edges cross-section

2. The air zone which reflects space between coils causes an increase in the number of FE. (It is aware because each section has different area and this produces different air zones)

3. The differences between the hexagon coil section and a circular section are significant.

As it is known, the memory limitations of the computer are due to the number of FE.

The most important conclusion is that most of the FE tools uses the same logic (mesh/resolution/checking the error estimator), and

\begin{tabular}{|l|l|}
\hline Section 1 & Section 2 \\
\hline & \\
\hline & \\
\hline & $\mathrm{R}_{1}, \mathrm{~A}_{1}, \mathrm{~A}_{2}$ \\
\hline
\end{tabular}

Figure 2: Consequently, a polygonal section coil can have the same resistance as the real coil in DC current.

\begin{tabular}{|l|l|l|l|l|}
\hline $\begin{array}{l}\text { Circular } \\
\text { Section }\end{array}$ & $\begin{array}{l}\text { Diamond } \\
\text { Section }\end{array}$ & $\begin{array}{l}\text { Outside } \\
\text { Square }\end{array}$ & $\begin{array}{l}\text { Inside } \\
\text { Square }\end{array}$ & $\begin{array}{l}\text { Hexagon } \\
\text { Section }\end{array}$ \\
\hline
\end{tabular}

Figure 3: Different coil sections. thus, imposed a determinate value of error estimator, independent of the mesh had been created; there is a reduction of FE changing the coil section [14].

From this point, the purpose of this study is to reduce the number of FE without losing accuracy.

\section{Approach Proposal}

In a comparison between a real 3D model and the model (coil with polygonal section), the results are not comparable, even at low frequency. One of the main reasons is the different cross-section of the coils (circle versus polygonal section).

One of the original contributions is to change the properties of the modelled material (usually copper) in order to have the same resistance as the actual coil at low frequency, regardless of the crosssectional shape (Figure 2).

\section{Coil Section Study}

It is necessary to choose the best polygonal section (less than 18 edges) that maintains the same accuracy in determining the resistance and inductance and minimizes the time needed to perform an FEA analysis.

The best approach is to compare several cross-sections. Figure 3 showed the used section.

The study consists of simulating several models without core and same coils (only changing the coil section shape).

After parameter extraction of the all simulations, it is possible to compare the resistance and inductance among models. In Figure 4 and Figure 5 are the resistance and inductance comparison.

As supported by these figures:

1. The best option is to use a hexagon cross-section because it best resembles the original circular cross-section.

2. The error in high frequency between a circle and hexagon

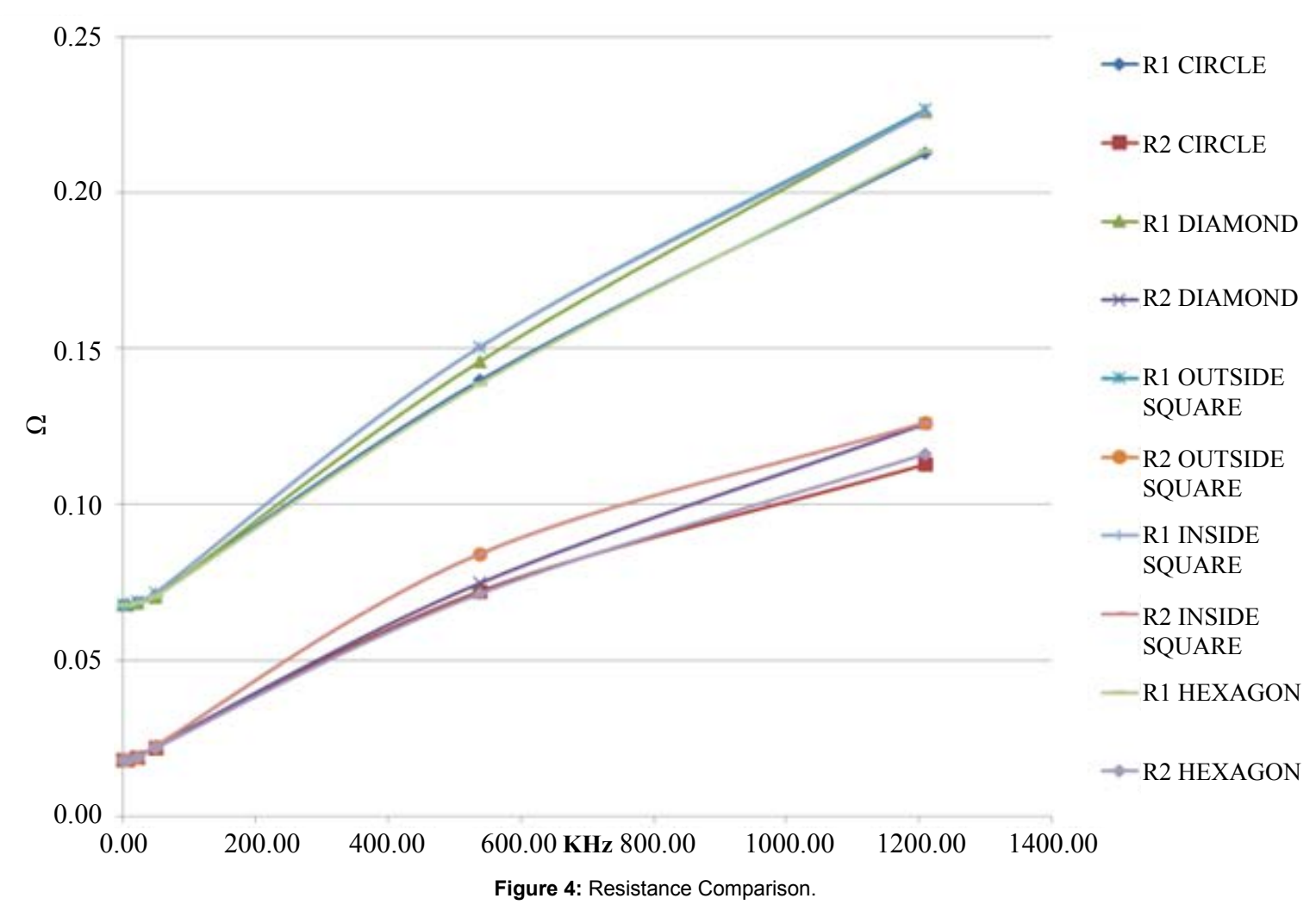




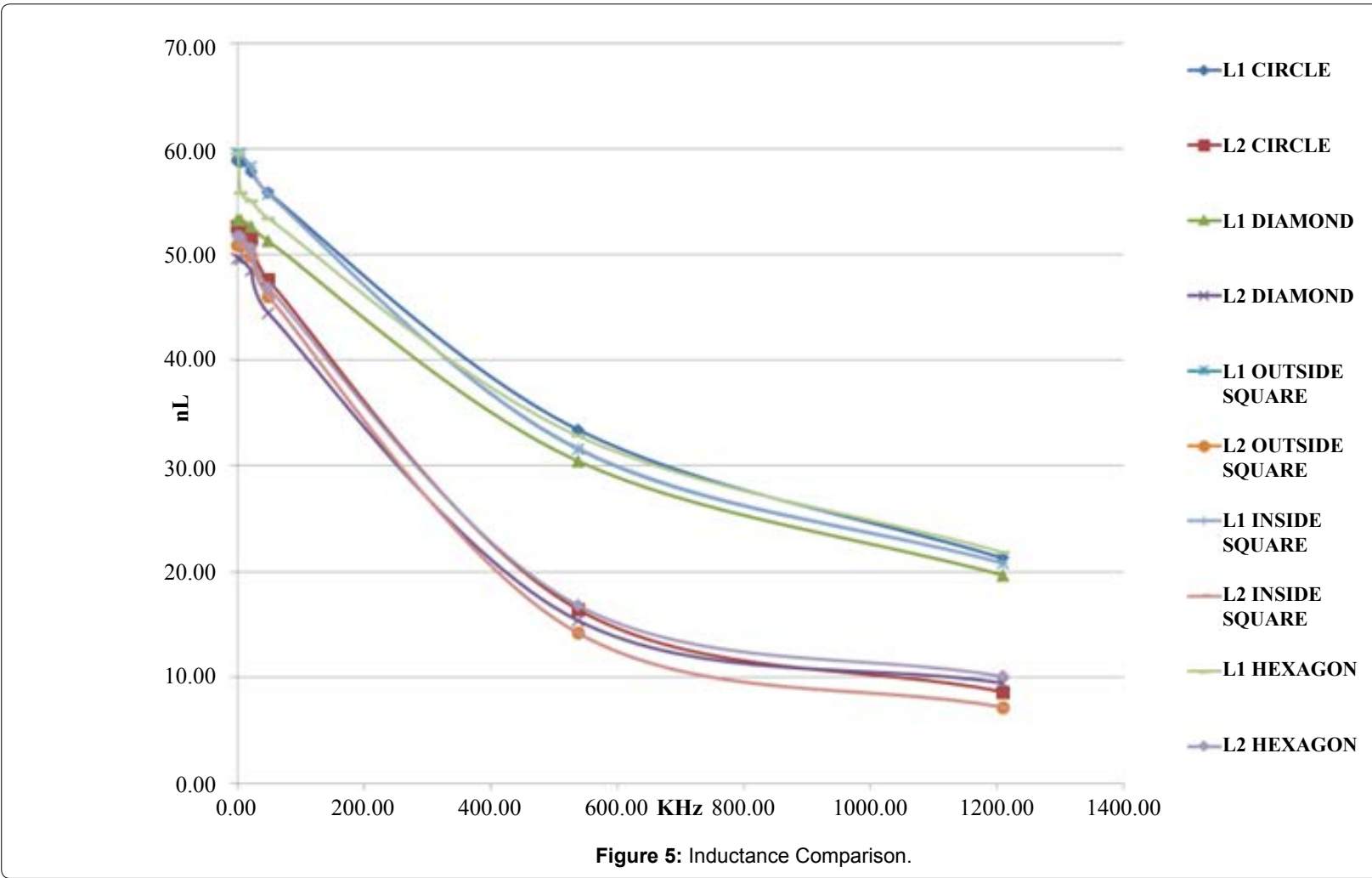

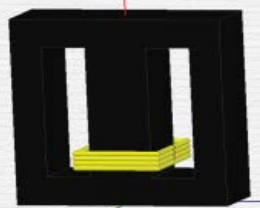

EE.42.21.15

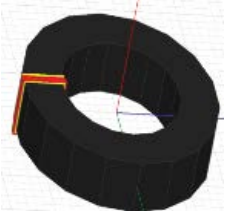

C107.65.25

\section{EE core (model EE. 42.21.15)}

\section{Toroidalcore (C107.65.25)}

Figure 6: Models.

Table 1: Information about skin effect.

\begin{tabular}{|l|l|l|l|}
\hline & AWG18 & AWG24 & Hz \\
\hline$r / \delta$ & 0,0234572 & 0,0120456 & 1 \\
\hline$r / \delta$ & 1,7199124 & 0,8831983 & 5376 \\
\hline$r / \delta$ & 3,4400648 & 1,7665197 & 21507 \\
\hline$r / \delta$ & 5,1601105 & 2,6497865 & 48391 \\
\hline$r / \delta$ & 17,200372 & 8,8326233 & 537678 \\
\hline$r / \delta$ & 25,800563 & 13,248938 & 1209776 \\
\hline
\end{tabular}

section is $0,4 \%$ for the resistance and $2,6 \%$ for the inductance

Thus, the choice for the coils is a hexagon cross-section.

The next step is to develop a study of simulations using the models in the following figure 6 .

These models have been chosen because EE \& Toroidal core are more common in transformers and power converters and their core does not have 3D symmetry. These models are a good choice because this study focuses on magnetic components that cannot be properly studied in $2 \mathrm{D}$.
These models will have a primary and a secondary.

- $\quad$ Primary: variable number of turns. AWG 18, copper

- Secondary: variable number of turns. AWG 24, copper

The selection of the coils has been arbitrary to establish a method to analyze models with polygonal sections in high frequency (Table 1).

Several simulations where only the number of coils varies (from one to four coils on each side) are done to compare the resistance and inductance between the real model and the model with hexagon cross-section.

For this study, many simulations are done, however only the most representative results are shown to understand the process and methodology. The following are examples of results using a Toroidal core (four turns in each coil).

The controlled parameters are the short circuit and the leakage impedance. The magnetizing impedance has not been used because it is independent of the coil cross-section shape.

The results Figure 7 and Figure 8 demonstrate that:

- Due to the material properties changes, in low frequencies, the resistive values are equal in every simulation.

- The precision of the inductance results are sufficient for this study. However, to increase the accuracy, the cross-section of the coil should be octagonal. For inductance, one of the most important factors is the distance between coils and turns themselves. The distances in an octagonal cross-section are more similar to an actual coil (circular) than a modelled hexagon section.

Changing the properties of the coil material suffices to model EE $\&$ Toroidal cores with hexagon coil cross-section for low frequencies.

\section{High Frequency Study}

The truncated model with a hexagon cross-section works in low frequencies but differs in medium and high frequencies. For this method, it is necessary to find a factor that modifies the resistance values to obtain similar values to the real coil. 


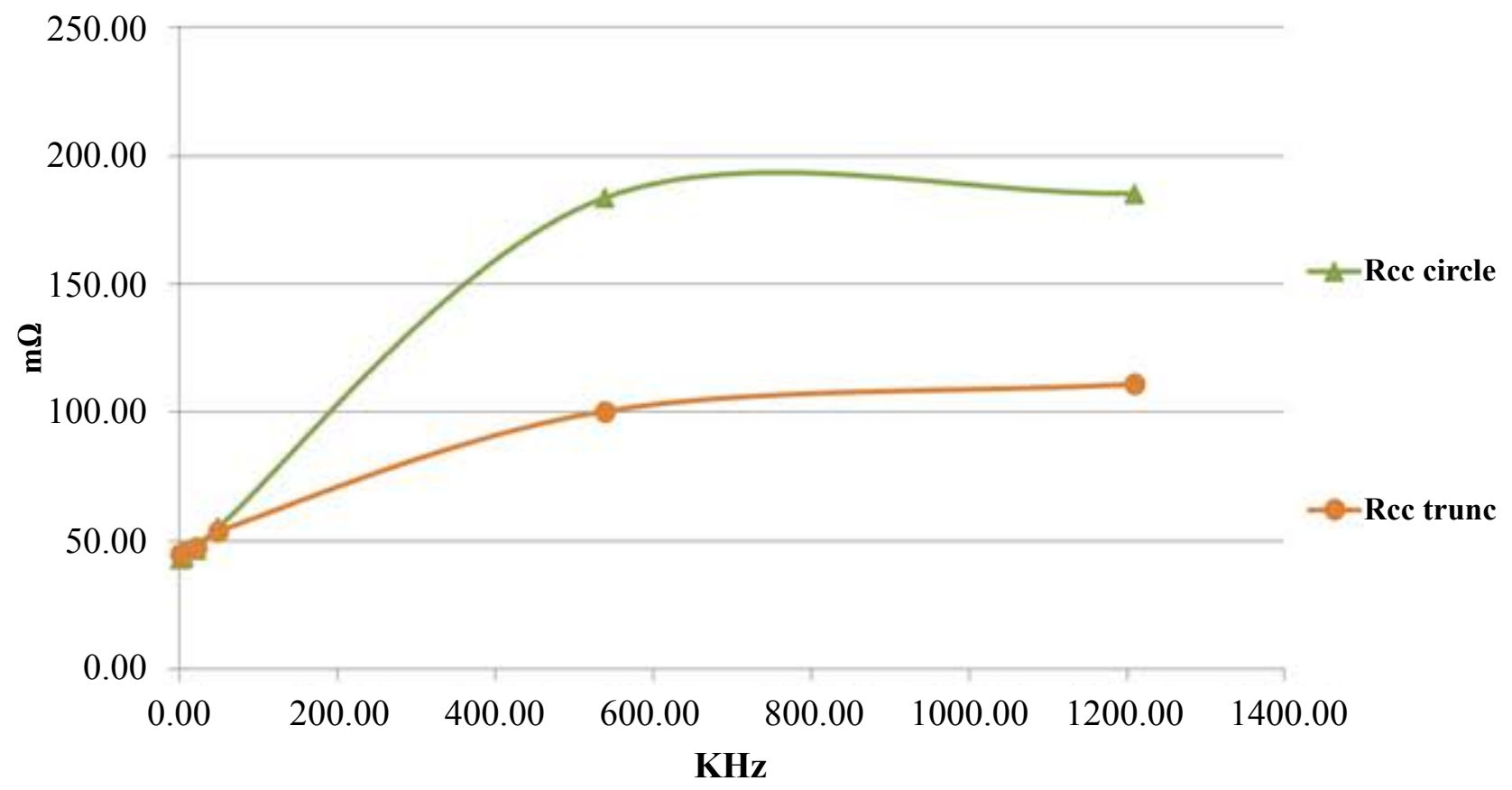

Figure 7: Resistance Comparison.

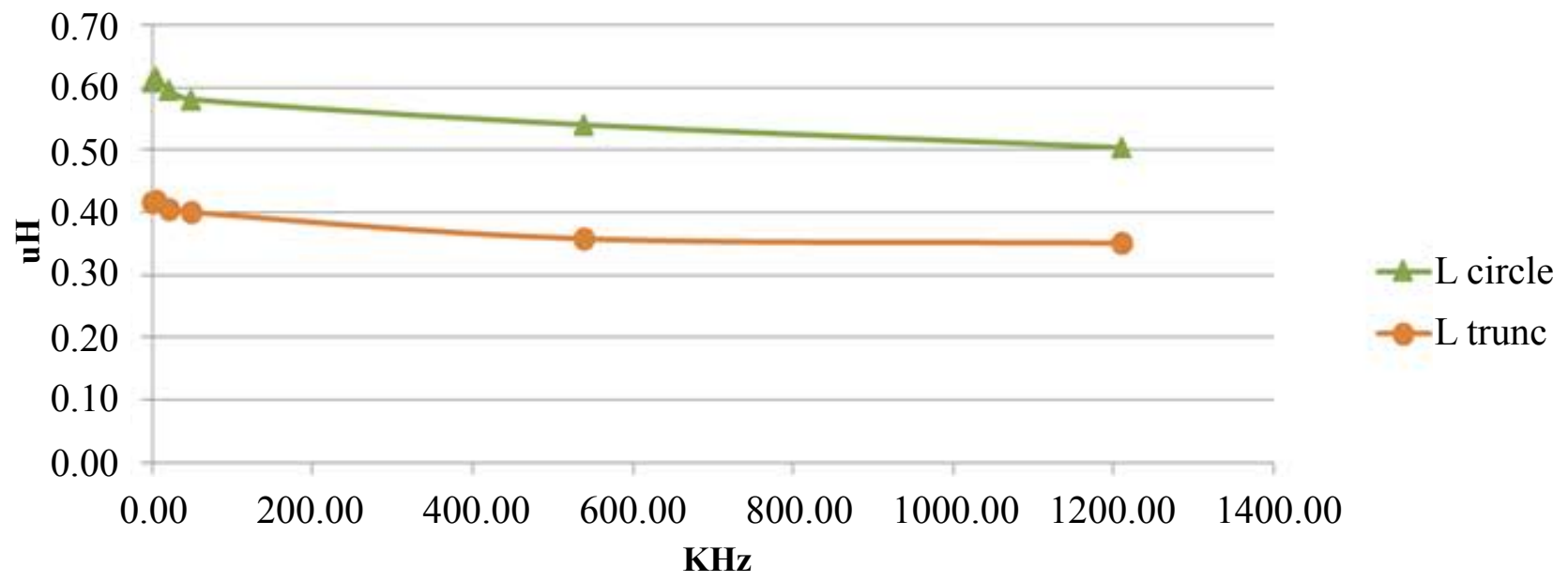

Figure 8: Inductance Comparison.

Table 2: The separated ranges.

\begin{tabular}{|l|l|}
\hline Lowfrequencies & $<20 \mathrm{KHz}$ \\
\hline Medium Frequencies & $20-50 \mathrm{KHz}$ \\
\hline High Frequencies & $<1,2 \mathrm{MHz}$ \\
\hline
\end{tabular}

To accomplish this, several frequency ranges are separated and the sought parameters are extracted to look for a linear behaviour (Table 2).

The next step in this study is to represent the difference between the truncated model and the real simulated model.

- $\quad$ EE core $(1,2,3,4,8$ turns in each coil)

- Toroidal core (1, 2, 3, 4 turns in each coil)

As is known, the relationship between resistance and frequency is of the $3^{\text {rd }}$ order. Thus, to find the linear behaviour in these ranges the plotted against the cubic frequency [15].

The next graphs (Figure 9, Figure 10 and Figure 11) show the results of this study for an EE core with eight turns in each side (also the slopes).

Using the method of this study, linear relationships are achieved in all studied cases:

- $\quad$ EE core (1, 2, 3, 4 and 8 turns)

- $\quad$ Toroidal core (1, 2, 3, 4 turns)

With this information, a table is built to compare the slopes with the number of turns for each core. This allows a linear relationship to be found for each frequency range for both models (EE \& Toroidal core).

\section{Method}

\section{Step I: Change the cross-section of the coils}

The cross-section of the coil is modelled as a hexagon crosssection inside the original circle of the coil. 

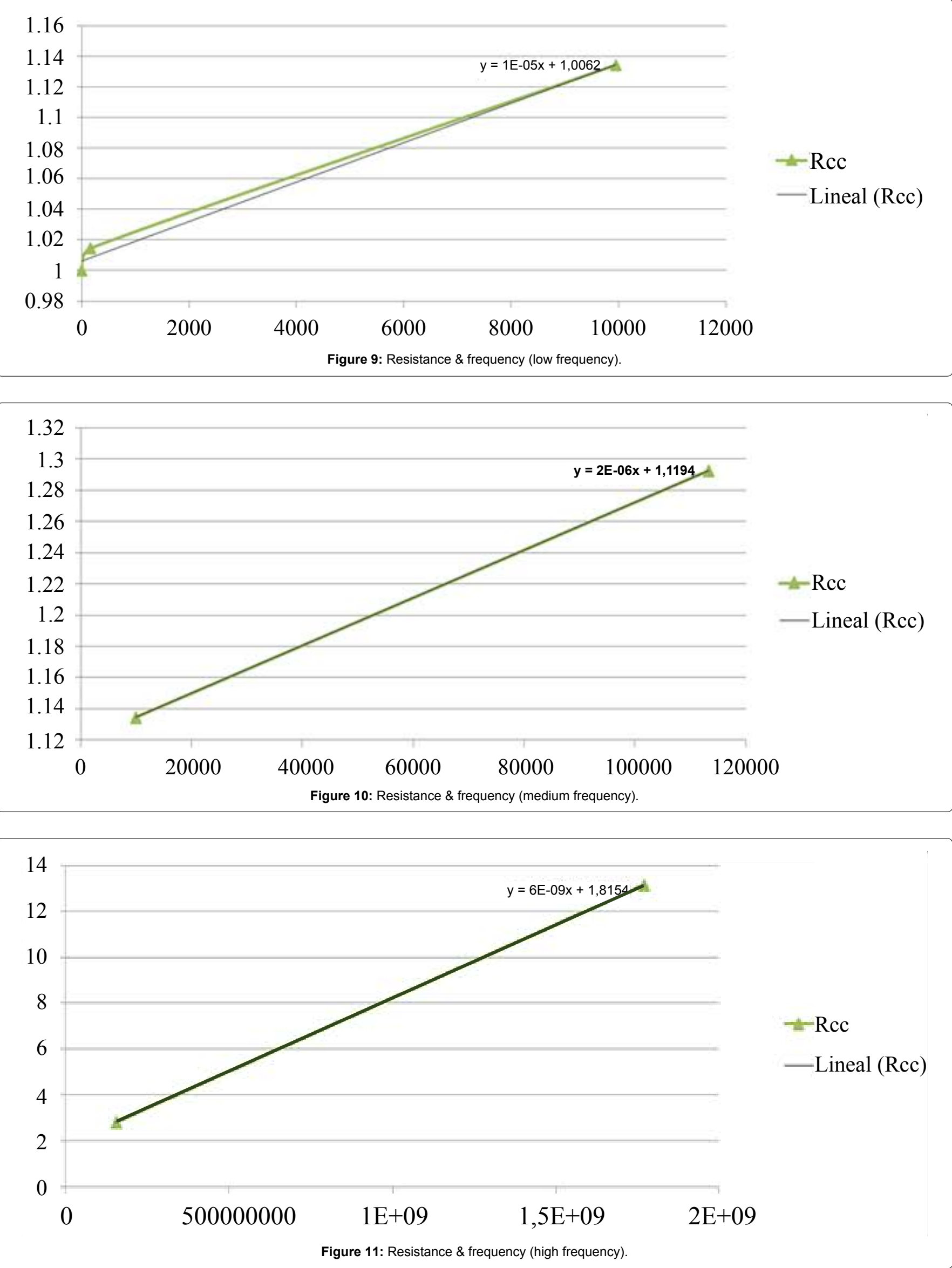

- The coil will be modelled as rings, not as a single spiralled coil. the same resistance as the real component in DC.

- The properties of the coil material will be changed to have Step II: Simulation of the component 


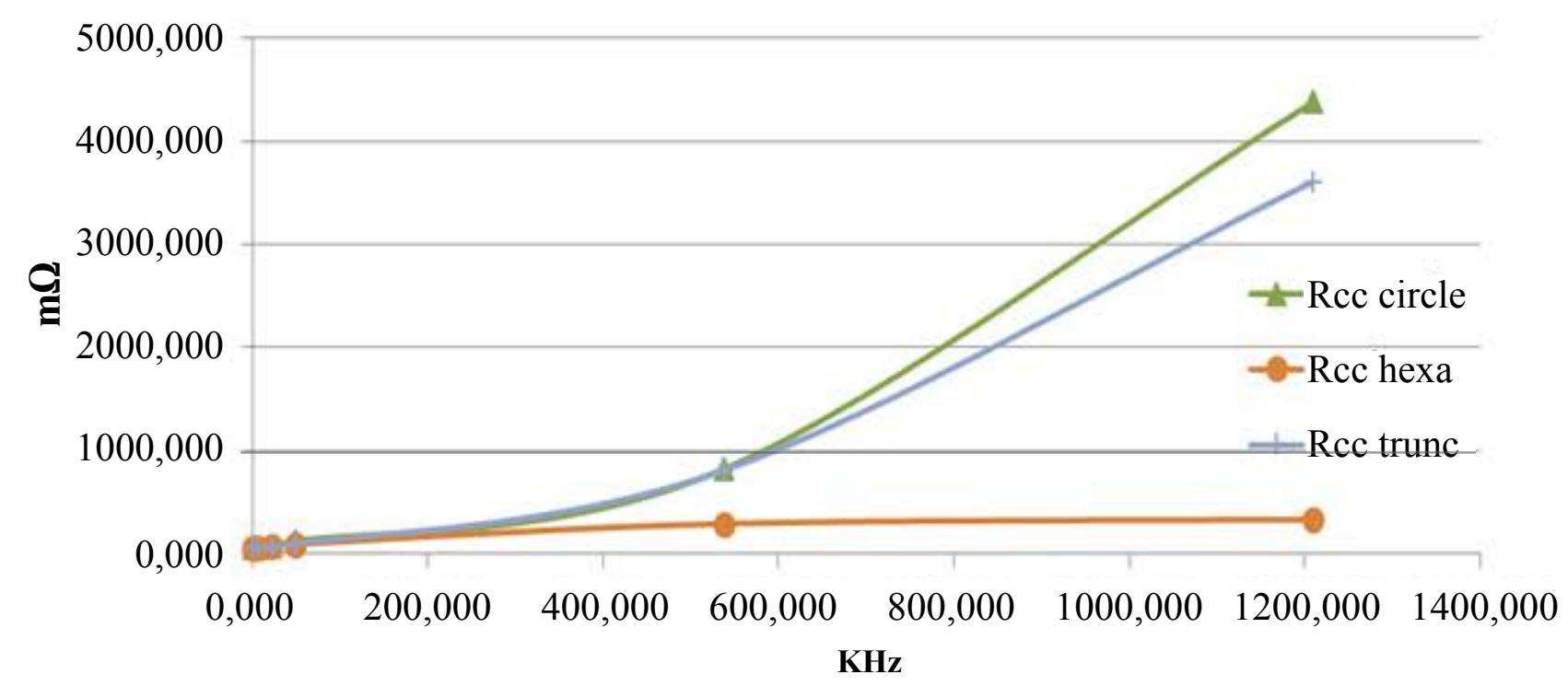

Figure 12: Rcc Comparison between models $<4 \%$.

Table 3: Correction factor.

\begin{tabular}{|l|l|}
\hline \multicolumn{2}{|c|}{ For an EE core } \\
\hline Medium Frequencies & $a=0,25 \times 10^{-15}$ \\
\hline High Frequencies & $\mathrm{a}=5 \times 10^{-18}$ \\
\hline \multicolumn{2}{|c|}{ For a Toroidal core } \\
\hline Medium Frequencies & $\mathrm{a}=0.1 / 10 \mathrm{E}-15$ \\
\hline High Frequencies & $\mathrm{a}=0.3 / 10 \mathrm{E}-18$ \\
\hline
\end{tabular}

Table 4: The proposed component was a transformer.

\begin{tabular}{|l|l|l|}
\hline Core & Coils & \\
\hline EE. 42.21.15 & Primary & Secondary \\
\hline & 8 turns, copper & 8 turns, copper \\
\hline Ferroxcube & AWG 18 & AWG 24 \\
\hline
\end{tabular}

- The post process will be done by the Application FEA with parameter extraction (energy analysis for multiwinding magnetic components [1]). In this case, the parameter extraction has been developed in 3D.

\section{Step III: Correlation for the resistance and inductance}

- The inductance is the same as the post-process $(<5 \%$ error, even in high frequencies)

- The resistance at Very Low Frequencies is the same as postprocess.

- The resistance at higher frequencies is determined using a correction factor (the second original contribution) as follows:

The correction factor, $y$, is as follows:

$y=a f^{3}$

(Table 3)

\section{Validation}

In order to validate the modeling process, several magnetic components were simulated. Two of them are presented here to illustrate the accuracy of the proposed method.

\section{Simulation \# 1}

The proposed component was a transformer with the following characteristics (Table 4)
Table 5: The real model simulation.

\begin{tabular}{|l|l|}
\hline Freq $\mathbf{( H z )}$ & Relative Error \% \\
\hline 5376 & 1,4 \\
\hline 537678 & 1,21 \\
\hline 1209776 & 17,6 \\
\hline
\end{tabular}

Table 6: The second model was of another transformer.

\begin{tabular}{|l|l|l|}
\hline Core & Coils \\
\hline C107.65.25 & Primary & Secondary \\
\hline & 3 turns, copper & 3 turns, copper \\
\hline Ferroxcube & AWG 18 & AWG 24 \\
\hline
\end{tabular}

Table 7: The real model needed 320898 FE and the truncated model 13480, FE, thus a less than $4,5 \%$ difference.

\begin{tabular}{|l|l|}
\hline Freq $\mathbf{( H z )}$ & Relative Error \% \\
\hline 5376 & 1,3 \\
\hline 537678 & 6,5 \\
\hline 1209776 & 9,5 \\
\hline
\end{tabular}

It compares the results of three simulations, the real model with a circular cross-section, the model with a hexagonal cross-section, and the model with changed properties for the coil and with the method applied.

The real model simulation had $811411 \mathrm{FE}$ and the truncated model only $30202 \mathrm{FE}$, so < 4\% (Figure 12, Table 5).

\section{Simulation \#2}

The second model was of another transformer with the following characteristics (Table 6):

Again, it is compared the results among the 3 models.

The real model needed $320898 \mathrm{FE}$ and the truncated model 13480 , FE, thus a less than $4,5 \%$ difference (Figure 13, Table 7).

\section{Conclusions}

The main conclusion of this study is a method to model any magnetic component (either EE core or Toroidal core) which maintains key parameters (Resistance \& Inductance), accuracy and reduces the amount of time to perform an FEA analysis.

Additionally, this study has found the following conclusions:

1) The main factor to increase the number of finite elements in a 


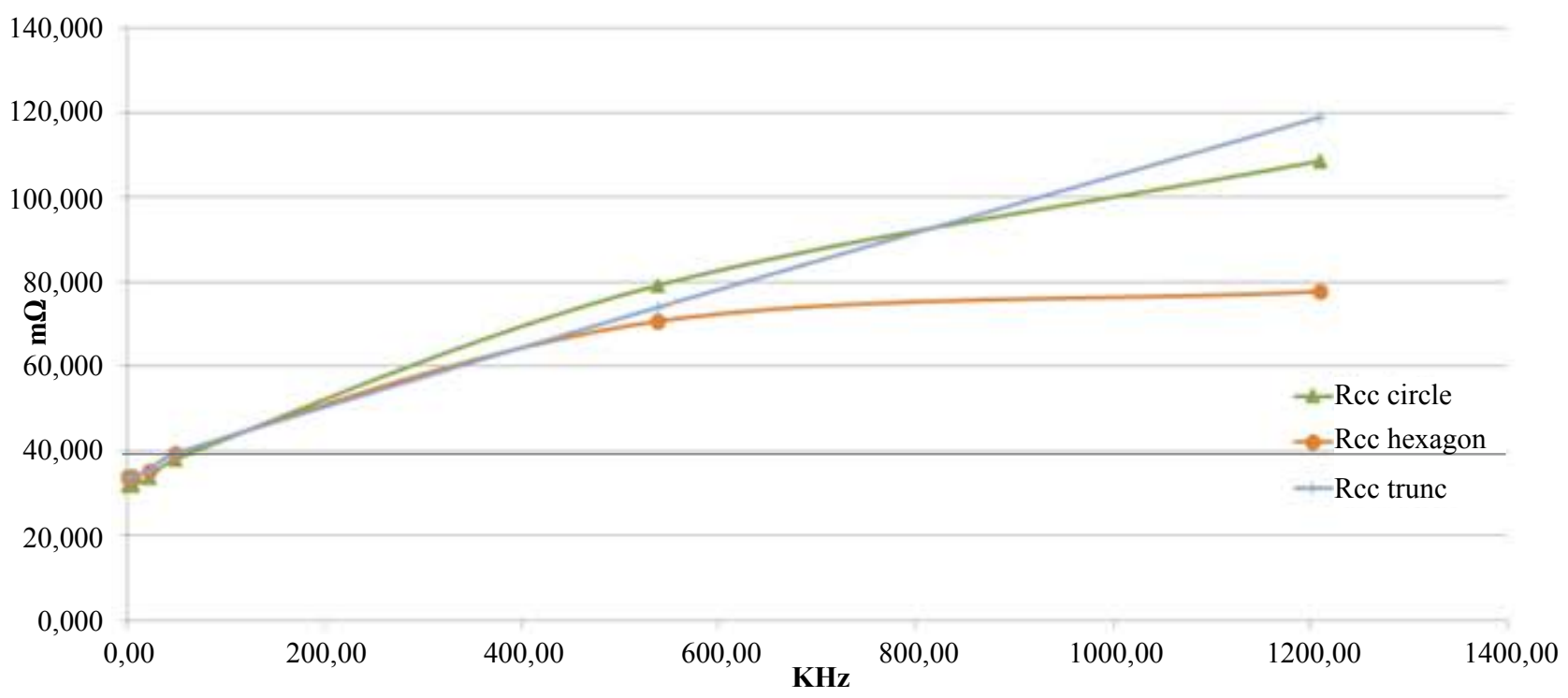

Figure 13: Rcc Comparison between models less than $4,5 \%$ difference.

magnetic component simulation is the number of turns and the coil cross-section.

2) By changing the properties of the copper, a polygonal crosssection inside the original circular cross-section can have the same resistance in DC current as the real coil. To model the behaviour of the inductance, this is a better approach when compared to a polygonal cross-section with the same area of the original circular cross-section.

3) The hexagon cross-section inside the coil is the best option when compared to a diamond or square cross-section.

4) For the purposes of this study, the values of inductance are very similar. To increase the accuracy of the model, it is necessary to increase the number of edges of the coil cross-section.

5) A correlation between resistance and frequencies is found when frequencies are separated into very Low, Medium and High ranges.

6) A method has been developed to obtain the parameters of resistance of inductance for transformers (EE core and Toroidal core) in low/medium and high frequency. This method is the most important original contribution in this publication.

\section{Future works}

With these conclusions in modelling and time computing for transformers, we can performance similar studies for other kind of transformers, it means with different core shapes. Even, this study could be developed for other magnetic components like coils.

Another different task would be to understand the effect of the shape coil, distance among them, etc for the inductance of the component.

\section{References}

1. Asensi R, Prieto R, Cobos JA, Uceda J (2007) Modeling High-Frequency Multiwinding Magnetic Components Using Finite-Elements Analysis. Transactions on Magnetics 43: 3840-3850.

2. Prieto R, Cobos JA, García O, Uceda J (1997) Interleaving techniques in magnetic components. APEC 2: 931-936.

3. García O, Cobos JA, Prieto R, Uceda J, Ollero S (1998) A standard design method for high frequency PCB Transformers. Telecommunications Energy Conference 1: 335-339.

4. Brauer BE, MacNeal LA, Larkin, Overbye VD (1991) New Method of Modeling Electronic circuits coupled with 3D Electromagnetic Finite Element Models.
Transactions on Magnetics 27: 4085-4088.

5. Piriou P, Razek (1992) A non-Linear Coupled 3D model for magnetic Field and Electric circuit Equations. Transactions on Magnetics 28: 1295-1298.

6. Lech Nowak, Krzystof Kowaski (1996) The 3D coupled Field-Circuit Simulation of Transients in Nonlinear Systems. Transactions on Magnetics 32: 1078-1081.

7. Slobodan Babic, Cevdet Akyel, Minya M Gavrilovic (2000) Calculation Improvement of 3D Liearn Magnetostatic Field Based on Fictitious Magnetic Surface Charge. Transactions on Magnetics 36: 3125-3127.

8. Hoke AF, Sullivan CR (2002) An Improved two-Dimensional Numerical modelling method for E-core Trasnformers. Applied Power Electronics Conference and Exposition 1: 151-157.

9. Prieto R, Østergaard L, Cobos JA, Uceda J (1999) Axisymmetric Modeling of 3D Magnetic Components. Applied Power Electronics Conference and Exposition 1: 213-219.

10. Lopera JM, Pernía AM, Díaz J, Alonso JM, Nuño F (1992) A complete Trasnformer Electric model, including frequency and geometry effects. Power Electronics Specialist Conference 2: 1247-1252.

11. Wilson PR (1998) Advanced Modeling and Simulation Tecchniques for Magnetic Components. Power Electronics and Variable Speed Drives 187 193.

12. Becker B, Grotstollen H, Heinemann L (1995) Computer Aided Design and Modeling of High Frequency Magnetic Components. Applied Power Electronics Conference and Exposition 1: 335-341.

13. Brauer JR, Schaefer SM, Lamert NJ, MacNeal BE (1999) Mixing 2D with 3D Finite Elements in Magnetic Models. Transactions on Magnetics 26: 21932195.

14. González JR, Prieto R (2014) Study of Limitations in Magnetic Components Modeling 3D. Seminario Annual de Automático, electronica industrial e instrumentation.

15. Belevitch V (1971) The lateral Skin effect in a flat conductor. Philips tech Rev 32: $221-231$. 